\title{
The universal structure of high-curvature regions of material lines in chaotic flows
}

\author{
A. LEONARD† \\ Graduate Aeronautical Laboratories, California Institute of Technology, Pasadena, CA 91125, USA
}

(Received 11 July 2008 and in revised form 2 October 2008)

Regions of high curvature of a material line as they evolve in a chaotic flow are considered. In such a region, the curvature as a function of arclength along the line is found to have a universal form with the peak curvature the only parameter involved. The alignment of the principal axes of the strain tensor with respect to the local tangent vector of the curve and the ratio of the two largest finite-time Lyapunov exponents play a key role. Numerical experiments with $\mathrm{ABC}$ flow demonstrate the result.

\section{Introduction}

We consider a passive line element as it evolves under the action of a chaotic flow and focus on regions of high curvature as they develop. The bending of material lines is, for example, relevant to the study of geometrical structures in turbulence and in the study of such structures in the magnetic field produced by a high-Prandtl-number kinematic dynamo (see e.g. Schekochihin et al. 2001).

In general, a differential element along the line will have a non-zero component aligned with the principal axis of maximum strain at some later time and will be stretched accordingly. At exceptional points along the line, the differential element will be orthogonal to the axis of maximum strain. It is at these points where the curvature may have a local maximum (Leonard 2005). The object of this paper is to determine the curvature as a function of arclength in the vicinity of these points of maximum curvature.

Indeed it has been observed in earlier studies that there is an anticorrelation of high curvature with stretch (Drummond \& Münch 1991; Hobbs \& Muzzio 1998). In his study of line evolution in two-dimensional model flows, Thiffeault (2004) determined that, in fact, there is a $-1 / 3$ power law relation between the magnitude of the stretch, $\eta$, and the curvature, $\kappa$, in regions of high curvature, i.e. $\kappa \eta^{3} \approx$ const. and he offered a theoretical explanation of this result. Leonard (2005) confirmed Thiffeault's result for three-dimensional line elements evolving in $\mathrm{ABC}$ flow and proposed an explanation based on the local alignment of the principal axes of strain with the tangent vector of the line. These considerations make up the starting point of the present investigation.

\section{Kinematics of passive line elements}

Consider the evolution of a material line or space curve $\boldsymbol{X}(q, t)$ parameterized by a material coordinate $q$ as it evolves in the velocity field $\boldsymbol{u}(\boldsymbol{x}, t)$. Thus,

$$
\frac{\partial \boldsymbol{X}}{\partial t}=\boldsymbol{u}(\boldsymbol{X}, t)
$$

$\dagger$ Email address for correspondence: tony@galcit.caltech.edu 
for all $q$. The tangent vector $\partial \boldsymbol{X} / \partial q=\boldsymbol{\ell}(q, t)$ satisfies

$$
\frac{\partial \boldsymbol{\ell}}{\partial t}=\frac{\partial \boldsymbol{u}}{\partial x_{j}} \ell_{j},
$$

where the velocity gradient tensor $\partial \boldsymbol{u} / \partial x_{j}$ is evaluated at the location $\boldsymbol{X}(q, t)$.

If $q$ is an arclength parameter at $t=0$, then $|\boldsymbol{\ell}(q, 0)|=1$ and

$$
\eta=|\ell(q, t)|
$$

is the local stretch of the curve at material point $q$. Let $\ell^{\prime}=\partial \ell / \partial q$. Then the curvature $\kappa$ of the space curve at $q$ is given by

$$
\kappa=\frac{\left|\boldsymbol{\ell} \times \boldsymbol{\ell}^{\prime}\right|}{|\boldsymbol{\ell}|^{3}} .
$$

\section{Properties of Lagrangian chaos}

Our objective is to characterize portions of the curve that have high curvature. For a velocity field that produces chaotic advection, the vector $\boldsymbol{\ell}$, satisfying (2.2), will in general experience exponential growth. We will require the deformation of the material surrounding particle $q$ accumulated since time $t=0$. Thus, if $\boldsymbol{F}(q, t)$ is the deformation tensor of interest then

$$
\frac{\partial \boldsymbol{F}}{\partial t}=\boldsymbol{U} \boldsymbol{F} \quad(\boldsymbol{F}(q, 0)=\boldsymbol{I}),
$$

where $U_{i j}=\partial u_{i} / \partial x_{j}$, and $\ell$ may be expressed as

$$
\boldsymbol{\ell}(q, t)=\boldsymbol{F} \ell(q, 0) .
$$

Let $\boldsymbol{F}$ have the polar decomposition

$$
\boldsymbol{F}=\boldsymbol{R}_{2} \boldsymbol{V},
$$

where $\boldsymbol{R}_{2}$ is a rotation matrix and $\boldsymbol{V}$ is positive-definite and is diagonalized by a rotation matrix $\boldsymbol{R}_{1}$ as follows:

$$
\boldsymbol{R}_{1}^{T} \boldsymbol{V R}_{1}=\mathrm{e}^{\lambda_{t}}=\left[\begin{array}{ccc}
\mathrm{e}^{\lambda_{1} t} & 0 & 0 \\
0 & \mathrm{e}^{\lambda_{2} t} & 0 \\
0 & 0 & \mathrm{e}^{\lambda_{3} t}
\end{array}\right]
$$

defining the finite-time Lyapunov exponents,

$$
\lambda_{i}=\lambda_{i}(X(q, 0), t)
$$

ordered so that

$$
\lambda_{1} \geqslant \lambda_{2} \geqslant \lambda_{3}
$$

Incompressibility $\left(U_{i, i}=0\right)$ yields the relation

$$
\lambda_{1}+\lambda_{2}+\lambda_{3}=0 .
$$

Note that, in general, $|\boldsymbol{\ell}|=\eta$ will increase as $\mathrm{e}^{\lambda_{1} t}$. However, there may be exceptional points, say $q=q_{0}$ where $|\boldsymbol{\ell}|$ will increase as $\mathrm{e}^{\lambda_{2} t}$. These points will be of particular interest to us. 

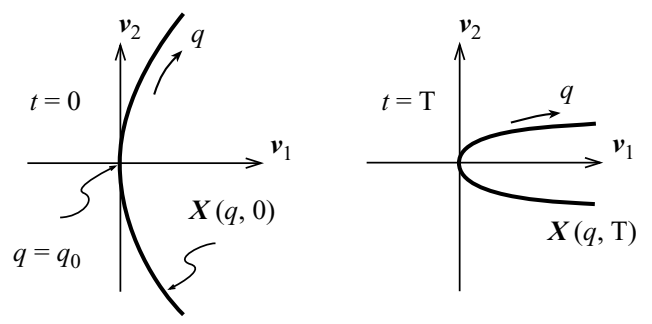

FIGURE 1. Deformation of the space curve $X(q, t)$ producing a region of high curvature. The tangent to the curve at $q=q_{0}$ is orthogonal to $v_{1}$.

\section{Application to material lines}

Here we follow closely Leonard (2005). Using the decomposition of $\boldsymbol{F}$ ((3.3) and (3.4)), we can express $\ell$ as follows:

$$
\ell=\sum_{i} \mathrm{e}^{\lambda_{i} t} a_{i} \boldsymbol{v}_{i},
$$

where $\boldsymbol{v}_{i}(i=1,2,3)$ are the orthonormal column vectors of $\boldsymbol{R}_{2} \boldsymbol{R}_{1}$.

In general, the right-hand side of (4.1) will be, as mentioned above, dominated by the term $i=1$. However, now consider $\ell$ as it varies along the space curve near a zero of $a_{1}$ at $q=q_{0}$. Note that

$$
\ell(q, t)=\boldsymbol{R}_{2} \boldsymbol{R}_{1} \mathrm{e}^{\lambda_{t}} \boldsymbol{R}_{1}^{T} \boldsymbol{\ell}(q, 0) .
$$

We consider four contributions to $\partial \ell / \partial q$ at $q=q_{0}$ corresponding to the partial derivatives of $\boldsymbol{R}_{2} \boldsymbol{R}_{1}, \mathrm{e}^{\lambda_{t}}, \boldsymbol{R}_{1}^{T}$ and $\boldsymbol{\ell}(q, 0)$, respectively. For our purposes, it is important to determine how these partial derivatives behave in terms of the factors $\mathrm{e}^{\lambda_{i} t}$. Fortunately, the asymptotic (in time) behaviour of the first three derivatives has been determined by Thiffeault (2002). The fourth derivative is just proportional to the curvature times the principal normal at $t=0$. Using $\S 4$ of Thiffeault (2002), we find that for $\lambda_{1}>2 \lambda_{2}$,

$$
\begin{aligned}
\boldsymbol{\ell}(q, t)=\left[\left(\hat{a}_{1}+\hat{\epsilon}_{1}\right)\left(q-q_{0}\right)\right] \mathrm{e}^{\lambda_{1} t} \boldsymbol{v}_{1}+\left[a_{2}+\left(\hat{a}_{2}+\hat{\epsilon}_{2}\right)\left(q-q_{0}\right)\right] \mathrm{e}^{\lambda_{2} t} \boldsymbol{v}_{2} \\
+\left[a_{3}+\hat{a}_{3}\left(q-q_{0}\right)\right] \mathrm{e}^{-\left(\lambda_{1}+\lambda_{2}\right) t} \boldsymbol{v}_{3}+\boldsymbol{O}\left(\left(q-q_{0}\right)^{2}\right),
\end{aligned}
$$

where $\hat{a}_{1}=O(1), \hat{a}_{2}=O\left(\max \left(1, \mathrm{e}^{\lambda_{2} t}\right)\right), \hat{a}_{3}=O\left(\max \left(1, \mathrm{e}^{\left(\lambda_{1}+3 \lambda_{2}\right) t}\right)\right), \hat{\epsilon}_{1}=O\left(\mathrm{e}^{2 \lambda_{2} t}\right)$ and $\hat{\epsilon_{2}}=$ $O\left(\max \left(\mathrm{e}^{-2\left(\lambda_{1}+2 \lambda_{2}\right) t}, \mathrm{e}^{-\left(\lambda_{1}+\lambda_{2}\right) t}\right)\right)$.

Keeping the leading order terms, we see that

$$
\boldsymbol{\ell} \approx \hat{a}_{1}\left(q-q_{0}\right) \mathrm{e}^{\lambda_{1} t} \boldsymbol{v}_{1}+a_{2} \mathrm{e}^{\lambda_{2} t} \boldsymbol{v}_{2}
$$

and

$$
|\boldsymbol{\ell}|^{2} \approx \hat{a}_{1}^{2}\left(q-q_{0}\right)^{2} \mathrm{e}^{2 \lambda_{1} t}+a_{2}^{2} \mathrm{e}^{2 \lambda_{2} t} .
$$

Note that $|\ell|$ is $O\left(\mathrm{e}^{\lambda_{1} t}\right)$ away from the zero $q_{0}$ but only $O\left(\mathrm{e}^{\lambda_{2} t}\right)$ at the zero - a dramatic change. A sketch depicting the deformation is shown in figure 1. A similar diagram was used by Schekochihin et al. (2004) in their study of folded structures of magnetic field lines in a turbulent flow. Note also that

$$
\left|\ell \times \ell^{\prime}\right| \approx\left|\hat{a}_{1} a_{2}\right| \mathrm{e}^{\left(\lambda_{1}+\lambda_{2}\right) t},
$$


so that from (2.4) the peak value of $\kappa=\kappa_{p}$ may be computed as

$$
\kappa_{p} \approx\left|\frac{\hat{a}_{1}}{a_{2}^{2}}\right| \mathrm{e}^{\left(\lambda_{1}-2 \lambda_{2}\right) t} .
$$

As noted, the above results (4.3)-(4.7) are valid for $\lambda_{1}>2 \lambda_{2}$. For $\lambda_{1}<2 \lambda_{2}$, we find, in general, that $\kappa_{p}=O(1)$. Perhaps it is worthwhile to note that some vortex models of inertial range turbulence also depend on $\lambda_{2} / \lambda_{1}$ (see e.g. Gilbert 1993; Leonard 2002).

\section{Universal structure}

In this section we derive an expression for the curvature distribution in the neighbourhood of a region of high curvature, which we shall henceforth denote by $\hat{\kappa}$. In terms of the parameter $r=\left(q-q_{0}\right) a_{2} \mathrm{e}^{\lambda_{2} t}$ we have, using (2.4), (4.5) and (4.6),

$$
\hat{\kappa}(r)=\frac{\kappa_{p}}{\left(\kappa_{p}^{2} r^{2}+1\right)^{3 / 2}},
$$

with

$$
\left|\frac{\partial \boldsymbol{X}}{\partial r}\right|=\frac{\mathrm{d} s}{\mathrm{~d} r}=\sqrt{\kappa_{p}^{2} r^{2}+1} .
$$

An expression for the function $\hat{\kappa}$ in terms of the arclength $s$ as measured from the point of peak curvature follows almost immediately. Letting $\kappa_{p} r=\hat{r}$ and integrating (5.2) we find,

$$
\kappa_{p} s=F(\hat{r})=\frac{1}{2}\left[\hat{r} \sqrt{\hat{r}^{2}+1}+\log \left(\hat{r}+\sqrt{\hat{r}^{2}+1}\right)\right] .
$$

Thus a universal form for $\kappa(s)$, depending only on a single parameter $\kappa_{p}$, is determined to be

$$
\hat{\kappa}(s)=\frac{\kappa_{p}}{\left(\left[F^{-1}\left(\kappa_{p} s\right)\right]^{2}+1\right)^{3 / 2}} .
$$

For small $\kappa_{p} s$ we find that

$$
\left.\left[F^{-1}\left(\kappa_{p} s\right)\right]^{2}=\left(\kappa_{p} s\right)^{2}-\frac{1}{6}\left(\kappa_{p} s\right)^{4}+\frac{11}{45}\left(\kappa_{p} s\right)^{6}+O\left(\left(\kappa_{p} s\right)^{8}\right)\right),
$$

and for large $\kappa_{p} s$,

$$
\left[F^{-1}\left(\kappa_{p} s\right)\right]^{2}=2 \kappa_{p} s-\frac{1}{2}\left(\log \left(8 \kappa_{p} s\right)+1\right)+O\left(\left(\log \left(\kappa_{p} s\right)\right)^{2} / \kappa_{p} s\right) .
$$

In the above development we have assumed that (4.5) and (4.6) hold in terms of $q$, the arclength parameter at $t=0$. However, it should be pointed out that it is sufficient that there exists a particular parameterization of the space curve such that (4.5) and (4.6) are valid. In fact any region of the curve that is locally parabolic will satisfy the universal form. See the discussion at the end of the next section.

\section{Numerical experiments - ABC flow}

As in our previous study (Leonard 2005) we use ABC flow (see e.g. Dombre et al. 1986) for our numerical experiments. If $\boldsymbol{u}=(u, v, w)$ then

$$
\left.\begin{array}{l}
u(x, y, z)=B \cos (y)+C \sin (z), \\
v(x, y, z)=A \sin (x)+C \cos (z) \\
w(x, y, z)=A \cos (x)+B \sin (y)
\end{array}\right\}
$$




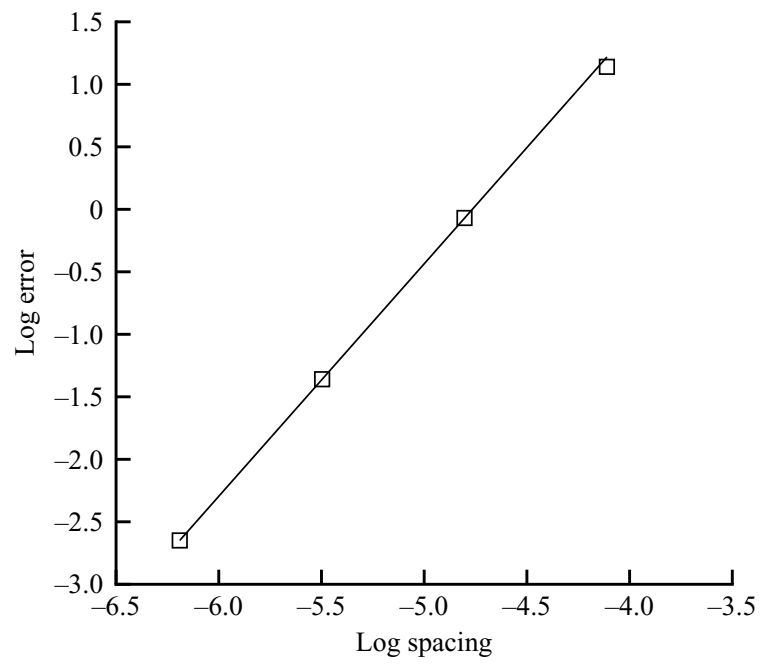

FIGURE 2. Error in $\kappa_{p}$ versus spacing between points for the case $\kappa_{p} \approx 40$ (symbols); line has slope $=1.86$.

We chose $A=5$ and $B=C=2$. This particular flow has no stagnation points but is known to produce extensive regions of chaotic particle motion and a Lyapunov exponent that is a maximum for the family of flows satisfying $B / A=C / A$ (Galanti, Sulem \& Pouquet 1992; Galloway \& O'Brian 1993).

The material line is initialized at $t=0$ as a circle of radius $R$ with a certain location and orientation and is discretized with $N_{0}$ equally spaced points. These points are moved according to (6.1) using third-order Runge-Kutta time integration with $\Delta t=0.01 / \sqrt{A^{2}+B^{2}+C^{2}}$. Every $n_{r}$ time steps, we remesh the curve using cubic spline interpolation such that consecutive knot points are equidistant with a distance equal to the separation at $t=0$. Use of cubic splines allows us to compute $\ell$ and $\ell^{\prime}$ with reasonable accuracy. We also use cubic splines to interpolate the stretch distribution along the curve, as this information must also be passed on to the new mesh.

For the following computations we used $N_{0}=6000, n_{r}=10$, and the initial circle was in the $z=0$ plane, centred at $x=y=0$, and had a radius $R=2$. The curve was evolved till $T \approx 2.08$ corresponding to 1197 time steps. The final number of points representing the curve is approximately 72000 . Runs were also performed at $N_{0}=2^{n} \times 750$ for $n=0,1$ and 2 to assure spatial convergence of the results. Figure 2 shows the convergence of the value of peak curvature for the high-curvature region shown in figure $5(d)$. Note that the convergence is nearly second-order in the distance between points along the curve. This is in accordance with the expected second-order accuracy of $\partial^{2} \boldsymbol{X} / \partial s^{2}$ when using cubic splines for interpolation. In each case, $\kappa_{p}$ and the corresponding location $s_{p}$ for a particular value of knot spacing were computed by using three pairs of $\left(\kappa_{i}, s_{i}\right)$, taken at the maximum value of $\kappa_{i}$ and the two on either side, and fitting these data to the expression (5.4) with $s$ replaced by $s-s_{p}$.

The curvature distributions are shown in figure 3 for times $T / 2$ and $T$ versus material coordinate. At $t=T$ there are seven regions of high curvature with $\kappa_{p} \geqslant 10$. Note that five of the seven high curvatures have developed to the point where $\kappa_{p} \geqslant 5$ at $t=T / 2$. The other two, appearing in $8.5<q<9$, have relatively small $\kappa_{p}$ values at $t=T / 2$, even though they have the first and third highest $\kappa_{p}$ values at $t=T$. 

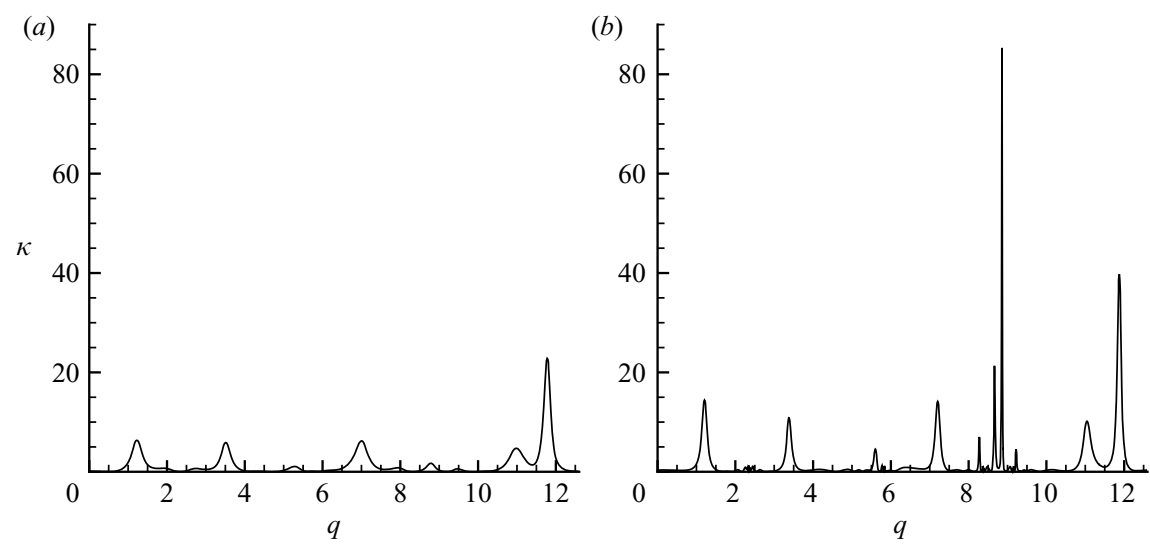

FIgURE 3. Curvature versus material coordinate $q$. (a) $t=T / 2$; (b) $t=T \approx 2.08$.
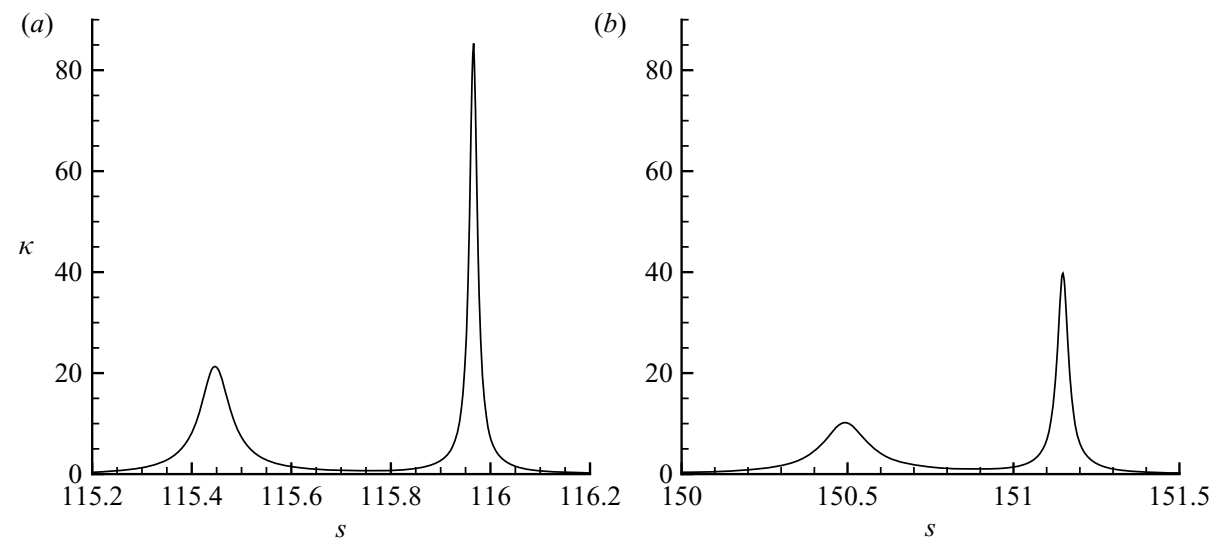

FIgURE 4. Curvature versus distance $s$ at $t=T:(a)$ third highest and highest $\kappa_{p}$; (b) seventh highest and second highest $\kappa_{p}$.

In figure 4 we show $\kappa$ versus distance parameter $s$ for the four high-curvature regions we will compare with the theory. They correspond approximately to $8.59<q<8.93$ (4a) and $10.67<q<12.26(4 b)$. Comparisons with theory ((5.4) with $s$ replaced by $s-s_{p}$ ) are shown in figure 5. Note that the agreement is very good, especially for the regions with the two highest $\kappa_{p}^{\prime} s$. Only the knot points are used in the graphics, both for $\kappa$ and $\hat{\kappa}$. This is noticeable particularly near the peak for the plots with the highest $\kappa_{p}$ (figure $5 b$ ).

Poincare maps were constructed for the material points corresponding to the highcurvature regions shown in figures 4 and 5 . They reveal that the line segments corresponding to $(a),(b)$ and $(c)$ of figure 5 develop high curvature in chaotic zones of the ABC flow while the high curvature of region $(d)$ develops in a zone of quasiperiodic orbits. In fact, it is not required that the flow be chaotic for high curvature to develop. A simple stagnation point flow will yield high curvature on a line that has non-zero initial curvature. A chaotic flow, however, will continue to produce new regions of high curvature as the material line is stretched.

In figure 6 we show the normalized difference between the actual curvature distribution and the theoretical distribution. Note that the differences for first and second highest peak regions are less than $1 \%$ of the peak values. For the region with 

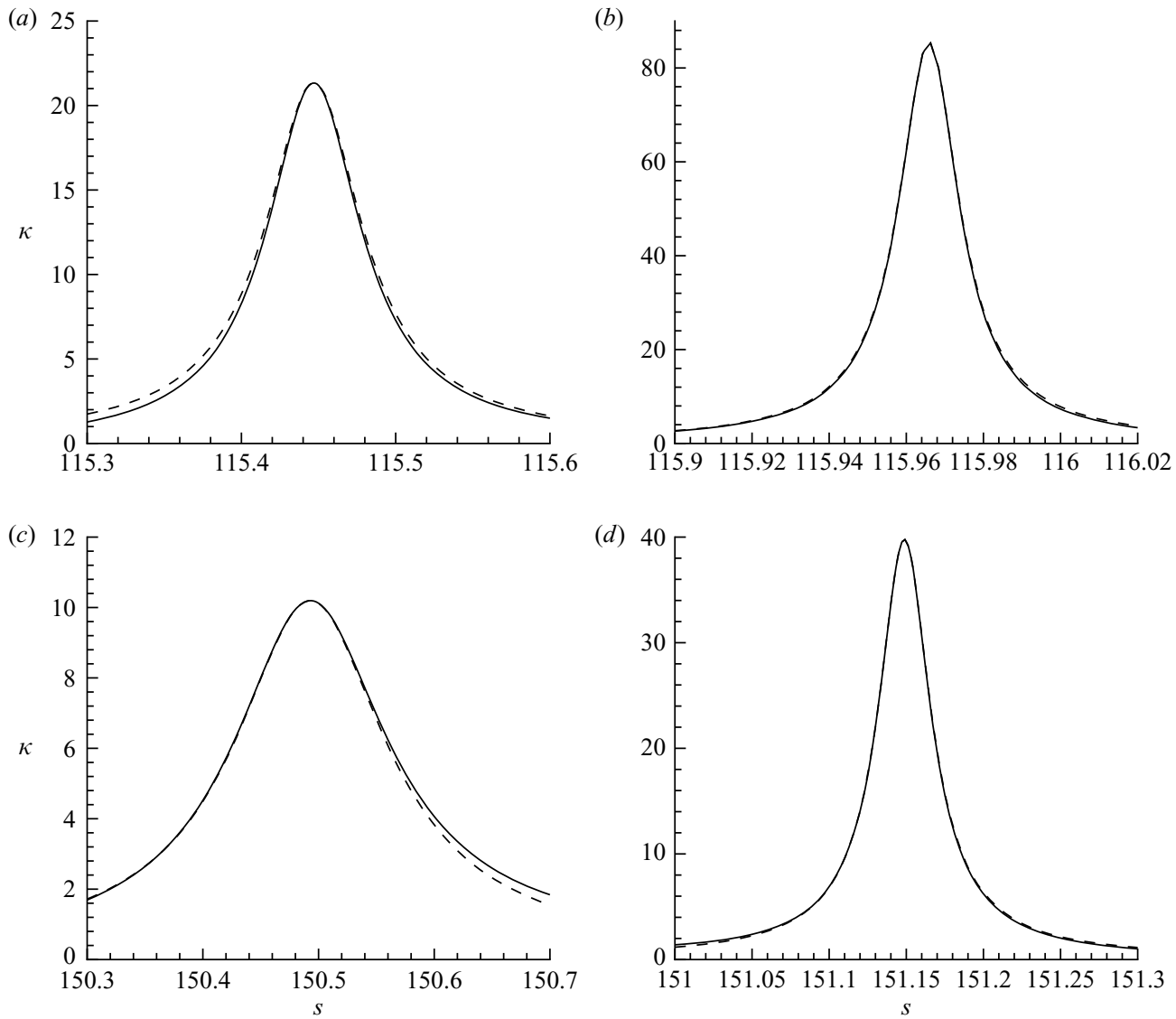

FIGURE 5. Curvature versus distance. $\kappa(s)$ (solid) and $\hat{\kappa}\left(s-s_{p}\right)$ (dashed) at $t=T$. (a) Third highest $\kappa_{p} ;(b)$ highest $\kappa_{p} ;(c)$ seventh highest $\kappa_{p} ;(d)$ second highest $\kappa_{p}$.

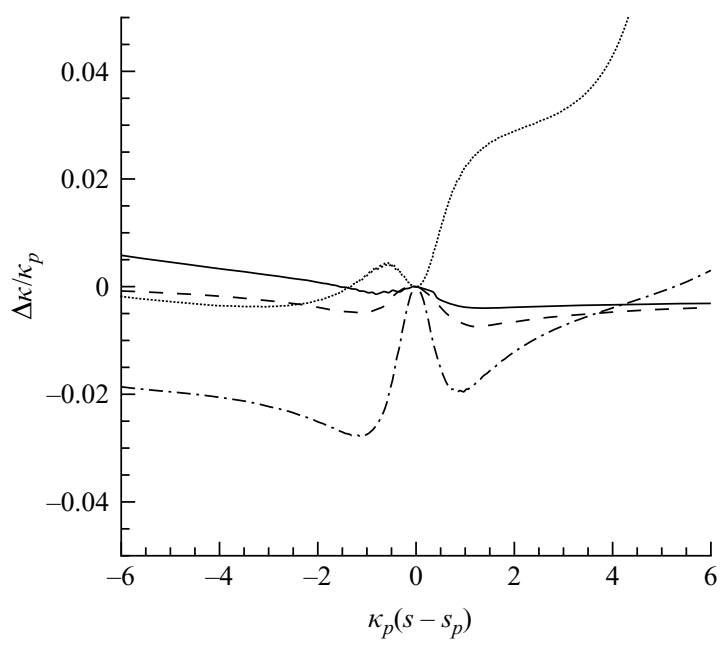

FiguRE 6. Non-dimensional difference in curvature versus non-dimensional distance for the regions shown in figure 5 with $\Delta \kappa=\kappa\left(s-s_{p}\right)-\hat{\kappa}(s)$ : dash-dot, third highest $\kappa_{p}$; dashed, highest $\kappa_{p} ;$ dotted, seventh highest $\kappa_{p} ;$ solid, second highest $\kappa_{p}$. 

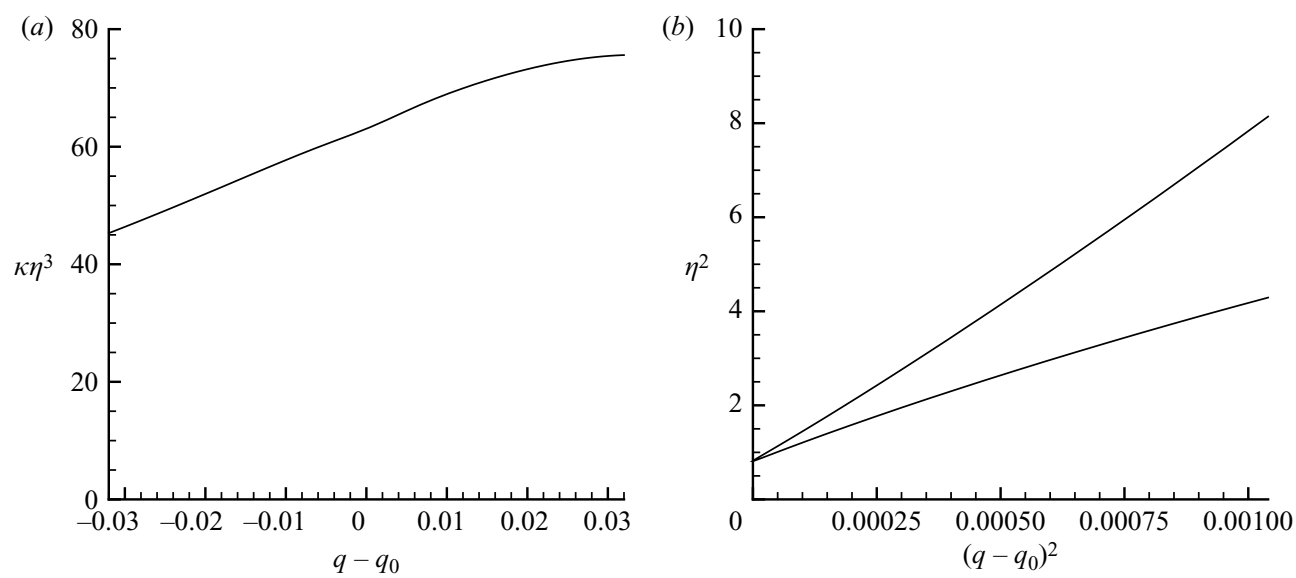

FigURE 7. (a) $\kappa \eta^{3}=\left|\partial \boldsymbol{X} / \partial q \times \partial^{2} \boldsymbol{X} / \partial q^{2}\right|$ versus $q-q_{0} ;(b) \eta^{2}=|\partial \boldsymbol{X} / \partial q|^{2}$ versus $\left(q-q_{0}\right)^{2}$.
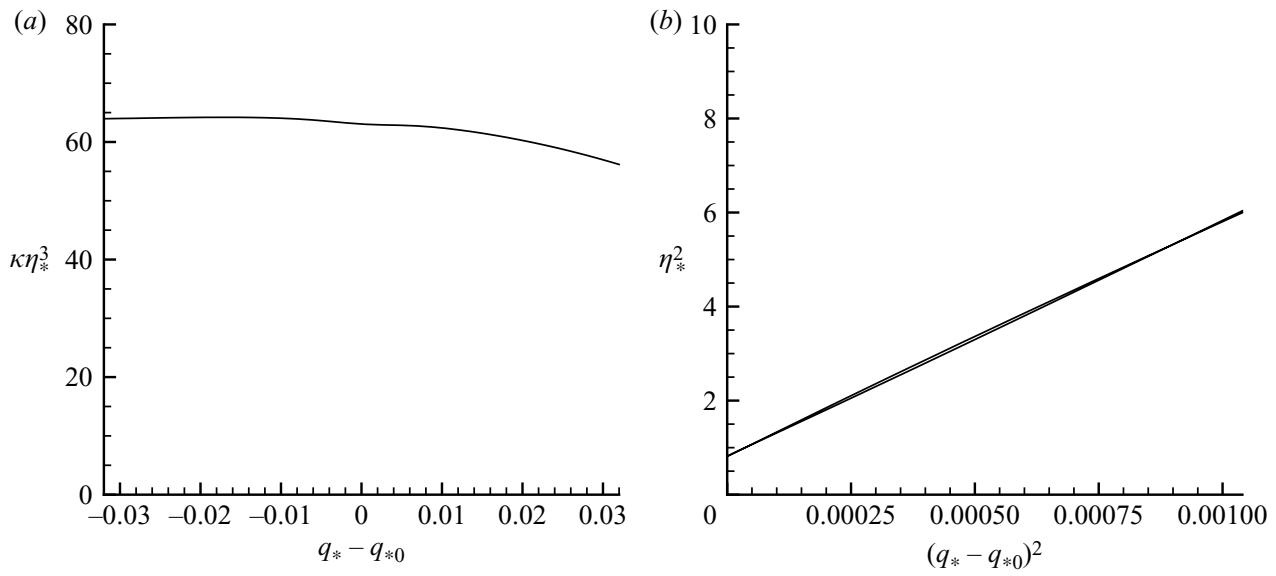

FIGURE 8 . Same as in figure 7 except with $q$ replaced by $q_{*}$.

the seventh highest peak (dotted), we need only to consider the domain $\left|\kappa_{p}\left(s-s_{p}\right)\right| \leqslant 2$ corresponding to the plot for this region in figure 5 . With this in mind, the differences for the remaining two regions are less than $3 \%$ of their respective peak values.

Figure 7 illustrates the point that the material coordinate $q$, that is also the arclength parameter at $t=0$, may not be optimal for the analysis presented in the previous section. Note that $\kappa \eta^{3}=\left|\ell \times \ell^{\prime}\right|$ is noticeably not a constant, in the vicinity of the high-curvature region of figure $5(b)$, as suggested by (4.6). Similarly, $|\ell|^{2}$ is not linear in $\left(q-q_{0}\right)^{2}$, i.e. both branches linear with the same slope, as claimed by (4.5). We found, in fact, that this portion of the curve underwent considerable non-uniform stretching before the deformation to high curvature took place, and that the simple transformation, $\mathrm{d} q_{*} / \mathrm{d} q=1+3.5\left(q-q_{0}\right)$ to a new parameter $q_{*}$ gives much agreement with (4.5) and (4.6) (see figure 8).

\section{Summary and discussion}

As a material line $X(q, t)$ with material coordinate $q$ evolves in time in a chaotic flow, a localized region of high curvature may develop around a point where the 
tangent vector $\boldsymbol{\ell}=\partial \boldsymbol{X} / \partial q$ is orthogonal to the principal axis of maximum strain. A necessary condition is that the largest finite-time Lyapunov exponent must exceed twice the second largest exponent. The strain tensor in question is that corresponding to the deformation starting at the time of initial development to some later time.

Our main result is that in such a region, the curvature as a function of distance from the point of peak curvature $\kappa_{p}$ is well approximated by $\kappa_{p}$ times a universal function of the distance times $\kappa_{p}$. The function itself is determined by analysis. The results are confirmed by numerical experiments in which material curves are evolved in $\mathrm{ABC}$ flow. Of course, the chosen ABC flow only has one length scale $\ell$ and one velocity scale $u$, both $O(10)$. Thus the time scale for the development of high curvature, $\ell / u$, in ABC flow is $O(1)$. However, the length scale of the high-curvature region is $O\left(1 / \kappa_{p}\right)$, which can be much smaller than and is not directly related to $\ell$. In three-dimensional turbulence, we also expect that the time scale for the development of high curvature to be $O(\ell / u)$ or, in this case, $O(1 / \omega)$, where $\omega$ is the r.m.s. vorticity. We again expect that the length scale of a high-curvature region will not be related to any flow length scale and has the potential to be smaller than the Kolmogorov length.

\section{REFERENCES}

Dombre, T., Frisch, U., Greene, J. M., Hénon, M., Mehr, A. \& Soward, A. M. 1986 Chaotic streamlines in the ABC flows. J. Fluid Mech. 167, 353-391.

Drummond, I. T. \& MüNCH, W. 1991 Distortion of line and surface elements in model turbulent flows. J. Fluid Mech. 225, 529-543.

Galanti, B., Sulem, P. L. \& Pouquet, A. 1992 Linear and non-linear dynamos associated with ABC flows. Geophys. Astrophys. Fluid Dyn. 66, 183-208.

Galloway, D. J. \& O’BRIAN, N. R. 1993 Numerical calculations of dynamics for ABC and related flows. In Solar and Planetary Dynamos (ed. M. R. E. Proctor, P. C. Matthews \& A. M. Rucklidge), pp. 105-113. Cambridge University Press.

GilberT, A. D. 1993 A cascade interpretation of Lundgren's stretched spiral vortex model for turbulent fine structure. Phys. Fluids A 5, 2831-2834.

Hobis, D. M. \& Muzzio, F. J. 1998 The curvature of material lines in a three-dimensional chaotic flow. Phys. Fluids 10, 1942-1952.

LeONARD, A. 2002 Interaction of localized packets of vorticity with turbulence. In Tubes, Sheets and Singularities in Fluid Dynamics (ed. K. Bajer \& H. K. Moffatt), pp. 201-210. Kluwer.

LeOnARD, A. 2005 Curvature and torsion of material lines in chaotic flows. Fluid Dyn. Res. 36, 261-275.

Schekochinin, A., Cowley, S., Maron, S. F., Taylor J. \& McWilliams, J. C. 2004 Simulations of the small-scale turbulent dynamo. Astrophys. J. 612, 276-307.

Schekochinin, A. A., Cowley, S. C., Maron, J. \& Malyshrin, L. 2001 Structure of small-scale magnetic fields in the kinematic dynamo theory. Phys. Rev. E 65, 016305:1-18.

Thiffeault, J.-L. 2002 Derivatives and constraints in chaotic flows: asymptotic behaviour and a numerical method. Physica D 172, 139-161.

Thiffeault, J.-L. 2004 Stretching and curvature along material lines in chaotic flows. Physica D 198, $169-181$. 